\title{
Initial Observations of Cell-Mediated Drug Delivery to the Deep Lung
}

\author{
Arun Kumar,* Mark Glaum,* Nagwa El-Badri, $†$ Shyam Mohapatra,* Edward Haller, $\$$ Seungjoo Park, $\neq$ \\ Leslie Patrick, $\neq$ Leigh Nattkemper,* Dawn Vo,* and Don F. Cameron $\neq$
}

\author{
*Department of Internal Medicine, University of South Florida College of Medicine, Tampa, FL, USA \\ $\dagger$ Department of Obstetrics \& Gynecology, University of South Florida College of Medicine, Tampa, FL, USA \\ ‡Department of Pathology \& Cell Biology, University of South Florida College of Medicine, Tampa, FL, USA
}

\begin{abstract}
Using current methodologies, drug delivery to small airways, terminal bronchioles, and alveoli (deep lung) is inefficient, especially to the lower lungs. Urgent lung pathologies such as acute respiratory distress syndrome (ARDS) and post-lung transplantation complications are difficult to treat, in part due to the methodological limitations in targeting the deep lung with high efficiency drug distribution to the site of pathology. To overcome drug delivery limitations inhibiting the optimization of deep lung therapy, isolated rat Sertoli cells preloaded with chitosan nanoparticles were use to obtain a high-density distribution and concentration $(92 \%)$ of the nanoparticles in the lungs of mice by way of the peripheral venous vasculature rather than the more commonly used pulmonary route. Additionally, Sertoli cells were preloaded with chitosan nanoparticles coupled with the anti-inflammatory compound curcumin and then injected intravenously into control or experimental mice with deep lung inflammation. By $24 \mathrm{~h}$ postinjection, most of the curcumin load ( 90\%) delivered in the injected Sertoli cells was present and distributed throughout the lungs, including the perialveloar sac area in the lower lungs. This was based on the high-density, positive quantification of both nanoparticles and curcumin in the lungs. There was a marked positive therapeutic effect achieved $24 \mathrm{~h}$ following curcumin treatment delivered by this Sertoli cell nanoparticle protocol (SNAP). Results identify a novel and efficient protocol for targeted delivery of drugs to the deep lung mediated by extratesticular Sertoli cells. Utilization of SNAP delivery may optimize drug therapy for conditions such as ARDS, status asthmaticus, pulmonary hypertension, lung cancer, and complications following lung transplantation where the use of high concentrations of anti-inflammatory drugs is desirable, but often limited by risks of systemic drug toxicity.
\end{abstract}

Key words: Sertoli cells; Drug delivery; Deep lung

\section{INTRODUCTION}

Drug delivery to diseased organs, such as the lungs, is challenging, especially when the delivery of the therapeutic agent is limited or when there is an urgent need to insure high-density, location-specific distribution of the drug. Pulmonary delivery of therapeutic drugs is a safe drug delivery route, especially for asthma and chronic obstructive pulmonary diseases (COPDs), and provides a number of benefits particularly with regard to absorption area and avoidance of first pass metabolism in the liver $(13,16,18,26)$. However, meeting the sustained drug release goal can be somewhat problematic $(14,16,18,26)$. The lungs tend to expel materials that are introduced via the airway and it is, therefore, difficult to keep the drug in the lung long enough for the sustained release to be effective when the key challenge is therapy intended to address pathology in the small airways, terminal bronchioles, and alveoli (i.e., deep lung), especially in the lower lungs. Additional challenges are associated with the elimination of excipients that sometimes contribute to toxicities.

Historically, aerosol formulations have not been able to transport medication to deep lung efficiently, particularly if there is severe obstruction from airway edema, mucus plugging, and/or interstitial edema. Metered dose inhalers (MDIs), breath-activated dry powder inhalers (DPIs), liquid jet, and ultrasonic nebulizers are not practical for the delivery of most macromolecules because of their low system efficiency, low drug mass per puff, poor formulation stability for macromolecules, and poor dosing reproducibility (4). The ideal particle size of inhaled medication should range from 1 to $3 \mu \mathrm{m}$ in diameter for optimal deposition efficiency to small airways 
and terminal bronchioles $(14,15)$. Particles smaller than this size may reach the alveoli but are quickly expelled during exhalation. Most existing aerosol systems, although effectively delivering the dispensed drug to the upper airways, only deliver a small percentage of the dispensed drug in the correct particle size for optimal deposition in the deep lung (23). Furthermore, the amount of drug deposited from the device is highly dependent on the patient's inhalation technique. The effective delivery of proteins and peptides is optimized only if the patient acquires the ability to inhale correctly (17).

Most aerosol systems today deliver a total amount of $<100 \mathrm{Cg}$ of drug per puff to the deep lung; this amount is too low to enable timely delivery of many macromolecules for the often required milligram-level doses $(7,15)$. Although recently there have been numerous positive developments advancing the effective delivery of drugs to the lungs by nanoparticles, the aggressive and efficient physiological defense system designed to rid the airway system of particulate and foreign matter is an ongoing obstacle to effective delivery of therapeutic nanoparticles to the lung, especially the deep lung (15).

A novel approach to deliver nanoparticles coupled with drugs to the deep lung is to utilize a biocompatible cell-based system to implement delivery by way of the peripheral vasculature instead of the pulmonary route. A successful protocol would optimize drug volume and delivery and more efficiently target the deep lung with high drug density distribution while avoiding the liver and the active protease and immunoprotective environment of the lung. Testis-derived Sertoli cells (SCs) may be an ideal cell type to accomplish these goals. Isolated Sertoli cells have been used for over a decade to facilitate cell transplantation therapy without themselves resulting in deleterious effects in the host $(8,19)$. They provide for the immunoprotection of allo- and xenogeneic cell transplants and avoid immune rejection by not succumbing to either cell- or antibody-mediated immune responses $(5,6,8,20,25)$.

One reported mechanism by which SCs immunoprotect cell grafts is by the induction of donor-specific tolerance in the host following the IV injection of xenogeneic SCs (19). In this protocol, the large SCs ( 30-50 $\mu \mathrm{m})$ appear to become entrapped in the precapillary pulmonary vascular bed where the cells are quickly cleared from the system without apparent detrimental effects to the individual. On the basis of this observation and as a means to optimize deep lung delivery of therapeutic drugs, we tested the capability of the aggressively phagocytic SCs to carry therapeutic nanoparticles to the pulmonary parenchyma by way of the peripheral venous vasculature and then the pulmonary arterial vasculature.

In this project we describe the delivery of chitosan nanoparticles (10) and chitosan nanoparticles coupled with curcumin, a naturally occurring anti-inflammatory compound, to the deep lungs in mice by preloading the nanoparticles into isolated SCs and then injecting these preloaded SCs into the lateral tail vein of the recipient mouse. To test the therapeutic effect of a drug delivered by the Sertoli cell nanoparticle (SNAP) protocol, ovalbumin (OVA) sensitized and challenged mice exhibiting pulmonary perivascular inflammation were treated with SNAP-delivered curcumin. We hypothesized that curcumin delivered in this way would quickly and efficiently produce a high concentration and broad distribution of the drug to the deep lung, resulting in an effective deep lung therapeutic event.

\section{MATERIALS AND METHODS}

\section{Preparation of Chitosan Nanoparticles}

Chitosan (CS, Vanson, Redmond, WA) molecular weight of $60,000 \mathrm{kDa}$ was used to prepare the nanoparticles by ionotropic gelation with negatively charged tripolyphosphatesodium (TPP) ions according to a previously reported procedure (27). Briefly, CS was dissolved in an aqueous solution of acetic acid at concentrations of $0.05,0.10,0.15$, and $0.20 \mathrm{wt} \%$. The concentration of acetic acid was 1.75 times as high as that of CS. For example, $0.10 \mathrm{wt} \% \mathrm{CS}$ solution was prepared in 0.175 wt $\%$ acetic acid solution. Then, $1.0 \mathrm{ml}$ of TPP solution was added drop wise to $0.5 \mathrm{ml}$ of CS solution in a $20-\mathrm{ml}$ glass vial under magnetic stirring at 600 (20 rpm measured by a tachometer using an octagonal stirring bars). The mixture was stirred for an additional $10 \mathrm{~min}$. The weight ratios of CS/TPP were $4: 1$, 4.5:1, 5:1, 5.5:1 and 6:1. At least five batches of CS NPs were obtained from each protocol.

\section{Labeling Chitosan Nanoparticle With FITC}

Dehydrated methanol $(100 \mathrm{ml})$, followed by $2.0 \mathrm{mg} / \mathrm{ml}$ of FITC (Fluorescein-C6-Amine Labeling Kit, PANVERA, Madison, WI) was added into $1 \% \mathrm{w} / \mathrm{v}$ chitosan hydrochloride in $0.1 \mathrm{M}$ acetic acid solution $(100 \mathrm{ml})$ with stirring at $1000 \mathrm{rpm}$ at ambient temperature. After $3 \mathrm{~h}$ of reaction in the dark at ambient temperature, the fluorescent-labeled chitosan was precipitated in $0.2 \mathrm{M} \mathrm{NaOH}$ and separated from unreacted label using dialysis membrane MWCO:12-14,000 with flat width of $45 \mathrm{~mm}$ with $1 / 15 \mathrm{M}$ phosphate buffer/0.2 $\mathrm{M} \mathrm{NaCl}(\mathrm{pH} 5.5)$ as elution solvent and dialyzed in $100 \mathrm{ml}$ of distilled water for 3 days in the dark (1). The labeled nanoparticles were characterized and used for uptake studies immediately upon preparation. TPP, acetic acid, FITC, chlorpromazine, filipin, sucrose, Triton X-100, and methanol were purchased from Sigma Chemical Co. (St. Louis, MO), and dialysis membrane MWCO:12-14,000 with flat width $45 \mathrm{~mm}$ was purchased from Spectrum Laboratories Inc. (Rancho Dominguez, CA). The transport me- 
dium was composed of Hanks balanced salt solution (HBSS), buffered with $10 \mathrm{mM} \mathrm{N}$-2-hydroxyethylpiperazine- $N$ '-2-ethanosulfonic acid (HEPES) and adjusted to pH 6.2 with $1.0 \mathrm{M} \mathrm{HCl}$ and purchased from Sigma.

\section{Sertoli Cell Isolation and Preparation}

Sertoli cells were isolated from prepubertal SpragueDawley rat testes by sequential enzymatic digestion as previously reported $(3,9)$ and maintained in DMEM/F12 supplemented with insulin-transferrin-selenium (ITS, $0.1 \%$, Sigma) and gentamicin sulfate $(50 \mathrm{mg} / \mathrm{ml}$, Sigma). Some SCs were tagged (i.e., prelabeled SCs) with either the DiO or DiI (Molecular Probes) fluorescent cell markers as specified by the company. Some SCs were incubated with FITC-labeled chitosan nanoparticles that were phagocytosed by the SCs (i.e., preloaded SCs). The nanoparticles $(500 \mu \mathrm{l}, 0.5 \mathrm{mg} / \mathrm{ml})$ were added directly to the medium and incubated with the SCs for $12 \mathrm{~h}$ and then aggressively washed (five times) with medium to remove nanoparticles not phagocytosed. All prelabeled and/or preloaded SCs were imaged utilizing the Olympus IX71 inverted florescent microscopy to verify nanoparticle phagocytosis and to define the fluorescent image profiles of the prelabeled and/or preloaded SCs.

\section{Project Design, Animals, Tissue Collection, and Morphological Evaluation}

The use of rats (to isolate SCs) and normal and OVAchallenged mice conformed to approved IACUC protocols for the animals. Rat SCs $\left(8 \times 10^{6}\right)$ preloaded with nanoparticles and/or prelabeled were injected into recipient normal mice (C57BL/6 and BALB/c mice, Harlan) through the lateral tail vein in a total volume $150 \mu \mathrm{l}$ culture medium following approved Institutional guidelines as previously reported (19). Tissues and organs were collected from SC-injected mice (experimental, $n=12$ ) and uninjected mice (controls, $n=4) 15 \mathrm{~min}, 1$ $\mathrm{h}$, and $24 \mathrm{~h}$ postinjection. Some mice were injected only with SCs prelabeled with either $\mathrm{DiO}$ (green when viewed through FITC filter) or DiI (red when viewed through the TRITC filter). Some mice were injected with only SCs preloaded with FITC-labeled nanoparticles (green when viewed through FITC filter). Some mice were injected with SCs preloaded with FITClabeled nanoparticles and then prelabeled with DiI (red when view through the TRITC filter and yellow when viewed through FITC filter). Organs and tissues collected included the lungs, spleen, thymus, liver, kidneys, pancreas, muscle, blood, and bone marrow. Some tissues were fixed with $4 \%$ paraformaldehyde/PBS for LM morphological evaluation, some with 3\% gluderaldehyde/PBS for TEM structural evaluation, some were flash frozen and cyosectioned for LM detection of fluorescence, and some unfixed whole organs were prepared for the detection of florescence utilizing the Olympus MVX10 florescence macroscope. Some tissues were processed for specific UV spectroscopic absorption assay (see below).

In addition to normal mice, some female C57BL/6 and BALB/c mice (Jackson Laboratory) were sensitized by IP injection of $10 \mu \mathrm{l}$ OVA with alum as previously described to mimic acute inflammatory allergic asthma $(22,24)$. The mice were challenged weekly for 3 weeks with an intranasal (IN) injection of $25 \mu \mathrm{l}$ OVA ( $20 \mathrm{mg} /$ $\mathrm{ml})$. One hour after the last IN challenge, the treatment group $(n=7)$ was injected via the tail vein with SCs $(8$ $\times 10^{6}$ ) preloaded with nanoparticles coupled with a 6.25 $\mathrm{mg} / \mu \mathrm{l}$ curcumin dose. Other OVA-challenged mice were not injected with SCs and served as untreated controls $(n=7)$. Lungs from treated (experimental) and untreated (control) OVA-challenged mice were collected $24 \mathrm{~h}$ postinjection, evaluated morphologically, and assayed for curcumin by specific UV absorbance assay (see below).

\section{Detection of Labels}

Fluorescent labels were determined by specific UV spectroscopic absorbance assay in SCs only and in tissues collected from experimental (SC injected) and control (uninjected) mice. Whole organs and tissues were homogenized in DI water, filtered, and the filtrated was use for the detection of labels at specific wave lengths and quantified by the absorbance assay. Absorbance values were obtained by UV spectrometry. Label detection for DiI prelabeled SCs (red) was performed at $553 \mathrm{~nm}$. Detection of SCs preloaded with FITC-labeled nanoparticles (green) was performed at $488 \mathrm{~nm}$ for FITC. SCs preloaded and prelabeled were assayed by specific UV absorption at wave lengths specific for the nanoparticle label and the SC label.

\section{Identification of Curcumin}

The specific UV absorbance value for curcumin was determined in SCs preloaded with curcumin-coupled nanoparticles as well as in the lungs from treated OVAchallenged mice (experimental, $n=7$ ) and untreated OVA-challenged mice (controls, $n=7$ ). The lungs were collected $24 \mathrm{~h}$ postinjection of the preloaded SCs $(8 \times$ $10^{6}$ ). The SCs and lungs were placed in labeled containers with $1 \mathrm{ml}$ PBS and then sonicated for $10 \mathrm{~s}$ by the Sonic Dismembrinator 60 (Fisher Scientific) to release the curcumin nanoparticles. The curcumin was then separated from the nanoparticles by adding $500 \mu \mathrm{l}$ DMSO to each container. The suspension then was centrifuged at $1200 \mathrm{rpm}$ for $10 \mathrm{~min}$ and the supernatant was collected. A UV spectrum was collected from each supernatant sample and the specific wave length absorption assay was performed at $420 \mathrm{~nm}$. 


\section{Data Presentation}

Means and SDs were derived from multiple UV absorbance assays values of FITC-labeled nanoparticles, curcumincoupled nanoparticles, prelabeled SCs $(8 \times$ $10^{6}$ ), and SCs preloaded with labeled nanoparticles. Absorbance assays for the various labels determined in mouse tissues were expressed as a percentage of the total amount of label deliver by the injected SCs by dividing the observed absorbance value by the absorbance value determined for the injected SCs $\left(8 \times 10^{6}\right)$.

\section{RESULTS}

\section{Microscopic Observations of Labeled Sertoli Cells and Image Definition}

Isolated rat SCs, in primary cell culture, aggressively and selectively phagocytosed FITC-labeled nanoparticles (i.e., preloaded SCs) as determined by labelspecific positive fluorescence (Fig. 1, green) in the SCs and the absence of fluorescence in control cells and contaminating peritubular cells (Fig. 1, 1a inset). At high magnification, single cell suspensions of preloaded SCs demonstrated positive cytoplasmic fluorescence for the FITC-labeled nanoparticles when viewed through the FITC filter (Fig. 1b, inset). When isolated SCs were prelabeled with $\mathrm{DiO}$ they imaged green when viewed through the FITC filter, both in vitro as well as in the lung (Fig. 2) and imaged red when prelabeled with DiI and view through the TRITC filter. When FITC-labeled nanoparticles were preloaded in SCs and then the SCs were prelabeled with DiI, these preloaded, prelabeled $\mathrm{SCs}$ in vitro imaged red when viewed through the
TRITC filter (Fig. 3a), but imaged yellow (Fig. 3b) when viewed through the FITC filter.

\section{Normal Mouse Lungs Following SC Injection}

Two outcome parameters were determined: microscopic observations and label detection.

Microscopy Observations. Prelabeled SCs were not evident in the mouse spleen, liver, thymus, kidney, muscle, pancreas, bone marrow, and blood $15 \mathrm{~min}$ or $1 \mathrm{~h}$ postinjection based on the absence of label-specific fluorescence in any of these tissues. The only tissue in which specific SC label was detected, microscopically, was the lung.

At 15 min postinjection, SCs prelabeled with $\mathrm{DiO}$ (green) were homogeneously distributed throughout the lung, including the lower lobes (Fig. 2), which at higher magnification appeared to be distributed in the precapillary vasculature around alveolar sacs (Fig. 2a, inset). SC label was not observed in the sham-injected control lung (Fig. 2c). SCs prelabeled with DiI and also preloaded with FITC-labeled nanoparticles were observed throughout the lungs and imaged red when viewed through the TRITC filter and yellow when view thought the FITC filter in cryosectioned lungs (Fig. 3c, d), as was the case for SCs in vitro (Fig. 3a, b). Cryosections from control lungs showed no specific label with either filter (Fig. 3c, d, insets).

At $1 \mathrm{~h}$ postinjection of preloaded, prelabeled SCs, unfixed lung cryosections exhibited some SC label (red) when view thought the TRITC filter (Fig. 3e), but there was no evidence of intact SCs as evidenced by the ab-

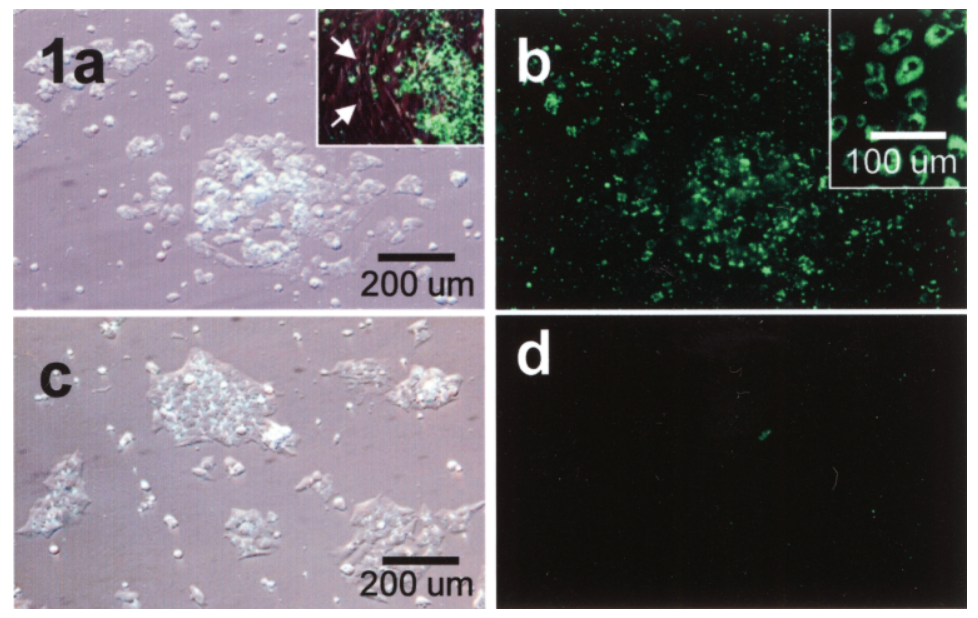

Figure 1. Isolated rat Sertoli cells (SCs) preloaded with FITC-labeled nanoparticles imaged by DIC (a, c) and fluorescence microscopy (b, d). SCs in (a, b) were incubated with the nanoparticles (green) and SCs in (c, d) were not. Inset: This culture of isolated SCs shows contaminating peritubular cells (arrows) that do not phagocytose the nanoparticles, indicating selective phagocytosis by SCs. Magnifications are the same in $(a-d)$. 

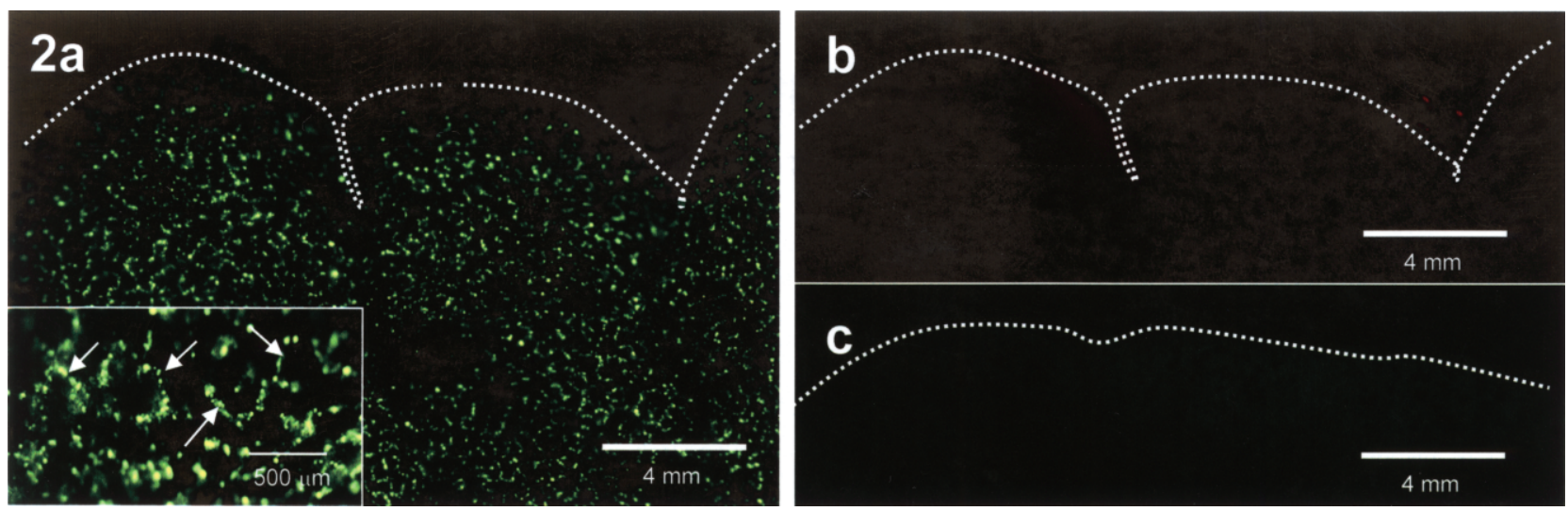

Figure 2. Unfixed whole mouse lung 15 min post-IV injection of DiO-labeled Sertoli cells (SCs). (a) View through the FITC filter, b) view through the TRITC filter, and c) unfixed control mouse lung view through the FITC filter. The homogeneous distribution of SCs label (green) throughout the lung is better appreciated at higher magnification (inset) showing to better advantage the distribution of prelabeled SCs (arrows) in what appears to be the perialveolar sac vasculature. When the lung is viewed through the TRITC filter (b), lack of positive florescence indicates that the florescence in (a) is label specific. The uninjected control mouse lung (c) shows no label-specific florescence when view through the FITC filter. Magnifications in (a-c) are the same.

sence of yellow fluorescent when viewed thought the FITC filter (Fig. 3f). There was, however, distinctive green fluorescence (Fig. 3f) when viewed through the FITC filter, indicating positive detection for the FITClabeled nanoparticles. Likewise, in unfixed whole lung samples, positive FITC label appeared homogeneously distributed throughout the lung when viewed at lower magnification by fluorescent stereoscopic microscopy (Fig. 3, Exp). As with the cyrosections, positive TRITC label was also evident (Fig. 3, Exp). Label was not detected by either filter in lung sections from control mice (Fig. 3, Cont). Tissue processed for electron microscopy from the $1 \mathrm{~h}$ postinjection lung exhibited small vessels and capillaries containing what appeared to be cell membrane debris and inclusions similar in appearance to aggregated labeled nanoparticles (Fig. 4, inset).

Label Detection. The number of preloaded and/or prelabeled SCs assayed $\left(8 \times 10^{6}\right)$ was the same as the number of SCs injected into the mouse host. The specific UV absorption in arbitrary units derived from SCs preloaded with FITC-labeled nanoparticles was $4.5 \pm$ 0.45 , from SCs prelabeled with DiI was $4.7 \pm 0.46$, and from SCs preloaded and prelabeled was $3.8 \pm 0.46$ and $3.7 \pm 0.46$, respectively.

At 15 min postinjection the mean specific UV absorbance of labeled nanoparticles in arbitrary units derived from mouse lung of preloaded SCs was $3.5 \pm 0.11$, from liver was $0.3 \pm 0.12$, and from the kidney was $0.2 \pm$ 0.12 . Specific UV absorbances in the spleen and lung from control mice were not detected (Fig. 5). When expressed as the percentage of the total nanoparticles delivered in the injected preloaded SCs, the lungs con- tained $92 \%$ of the total load, while the liver and the kidneys contained $8 \%$ and $5 \%$, respectively.

At $1 \mathrm{~h}$ postinjection, the mean specific absorbance of labeled nanoparticles from the mouse lung of preloaded SCs was $2.5 \pm 0.12$, from liver was $0.4 \pm 0.12$, and from kidney was $0.2 \pm 0.10$. Specific UV absorbance of labels from the spleen and lungs from control mice were not detected (Fig. 5). When expressed as the percentage of the total nanoparticles delivered in the injected preloaded SCs, the lungs contained $65 \%$ of the total load, while the liver and the kidneys contained $10 \%$ and $5 \%$, respectively (Fig. 6).

\section{OVA-Challenged Mouse Lungs Following SC Injection}

Curcumin-coupled nanoparticles were preloaded in SCs $\left(8 \times 10^{6}\right)$ and then delivered to the mouse lungs by injection via the lateral tail vein. Lungs from SNAPtreated and untreated OVA-challenged were collected 24 $\mathrm{h}$ postinjection.

Microscopic Observations. Lung sections from all seven untreated OVA-challenged control mice presented with diffuse perivascular inflammatory cell invasion into the interstitial pulmonary space (Fig. 7a), as previously described (11). However, lung sections from all seven OVA-challenged mice injected with SCs preloaded with curcumin-coupled nanoparticles were clear of inflammatory pathology $24 \mathrm{~h}$ postinjection as judged by the absence of inflammatory cell types in the pulmonary tissue (Fig. 7b).

Label Detection. Curcumin was assayed in preloaded SCs $\left(8 \times 10^{6}\right)$ in vitro and also assayed in lungs collected from the OVA-challenged mice $24 \mathrm{~h}$ postinjection of the 

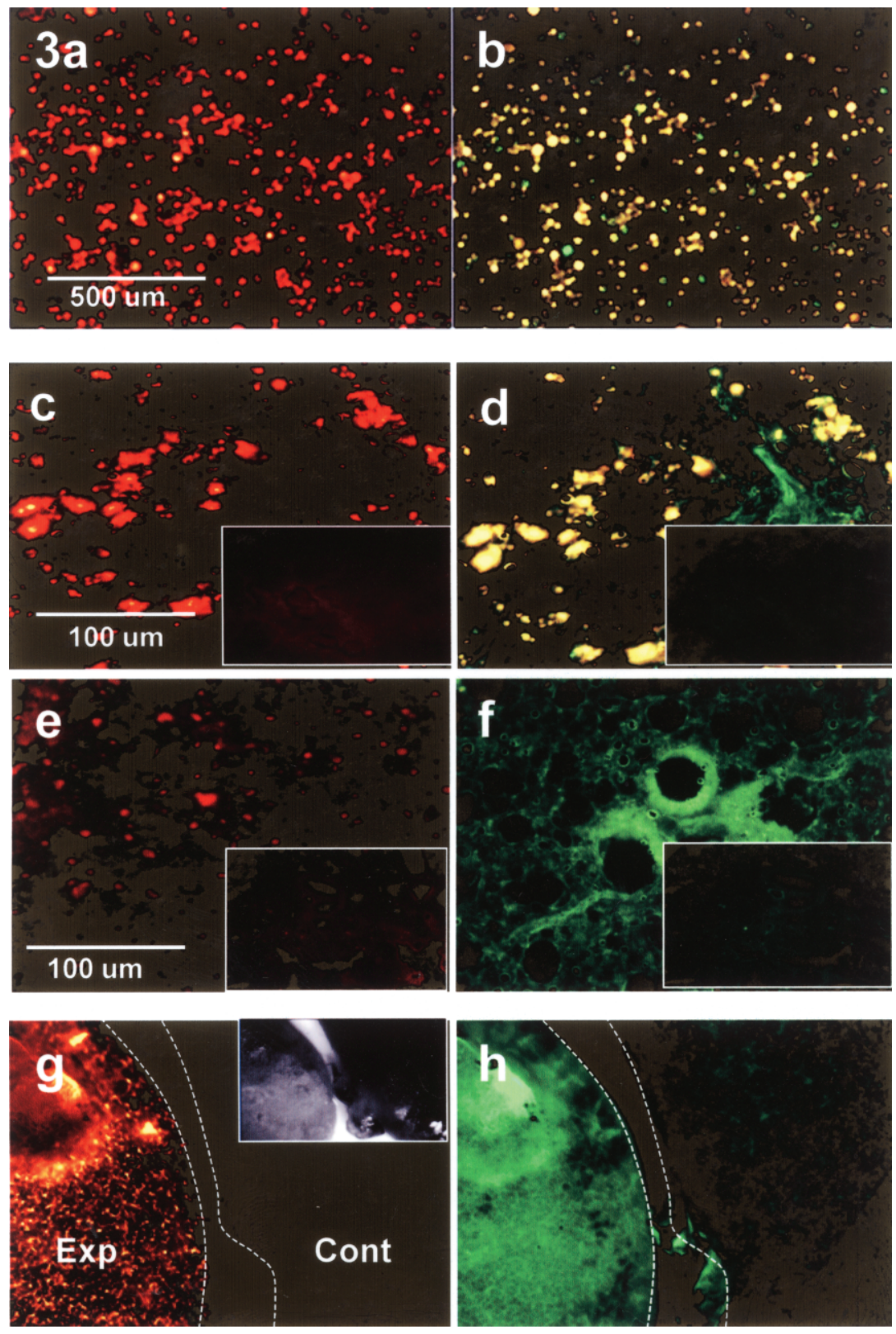

Figure 3. Sertoli cells (SCs) preloaded with FITC-labeled nanoparticles and prelabeled with DiI viewed through the TRITC filter (a, c, e, g) and through the FITC filter (b, d, f, h). Intact cells in vitro image red through the TRITC filter (a) and yellow through the FITC filter (b). Intact SCs in lung cryosections 15 min postinjection (c, d) image identically to the cells in vitro. However, at 1 $\mathrm{h}$ postinjection $(\mathrm{e}, \mathrm{f}$ ) note the absence of intact SCs (yellow) when viewed through the FITC filter but positive fluorescence for FITC-labeled nanoparticles (green). Cryosections from uninjected control mice (insets) show no positive fluorescence for SCs or nanoparticles. Low magnification macroscopic images of whole lung samples from mice $1 \mathrm{~h}$ postinjection $(\mathrm{g}, \mathrm{h}$ Exp) imaged similarly to the sectioned lungs $1 \mathrm{~h}$ postinjection $(\mathrm{e}, \mathrm{f})$. SC label was present when viewed through the TRITC filter (g, Exp), but the absence of overlap fluorescence (yellow), when viewed through the FITC filter (h, Exp), indicated the absence of intact SCs but instead the presence of labeled nanoparticles (green). Whole lung samples from control mice (g, h Cont) showed no positive fluorescence. Inset in (g) shows the two whole lung samples (Exp and Cont) imaged by bright field stereoscopic microscopy. 


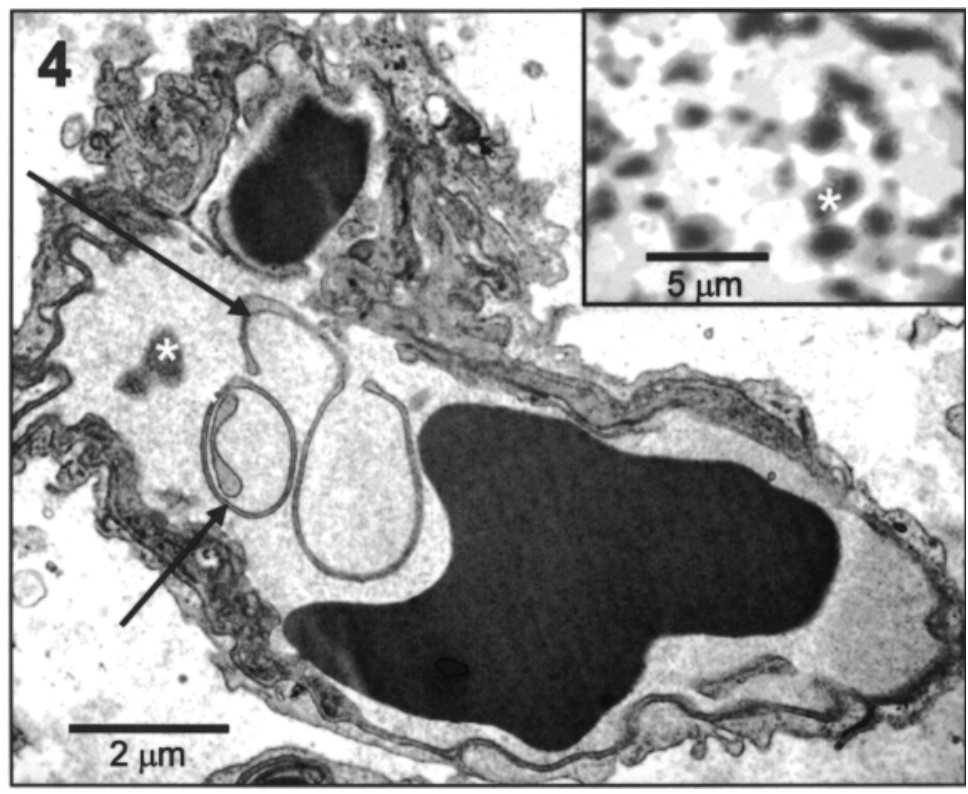

Figure 4. Electron micrograph of mouse lung $1 \mathrm{~h}$ postinjection of preloaded, prelabeled rat Sertoli cells. As illustrated in this image, pulmonary capillaries in the lungs of injected mice contained cellular debris (arrows) and intracapillary densities (asterisk) similar in appearance to aggregated labeled nanoparticles. Inset: Electron micrograph of aggregated labeled nanoparticles (asterisk).

preloaded SCs. The mean specific wave length absorbance from the preloaded SCs in vitro was $4.28 \pm 0.46$ and from the lungs of injected OVA-challenged mice $(n=7)$ was $3.72 \pm 0.72$. Curcumin was not detected by specific wave length absorbance in the lungs of unin- jected OVA-challenged mice $(n=7)$. When expressed as the percentage of the total amount of delivered curcumin (curcumin in the injected SCs), the lungs contained $\sim 90 \%$ of the total amount of curcumin injected (Fig. 6).

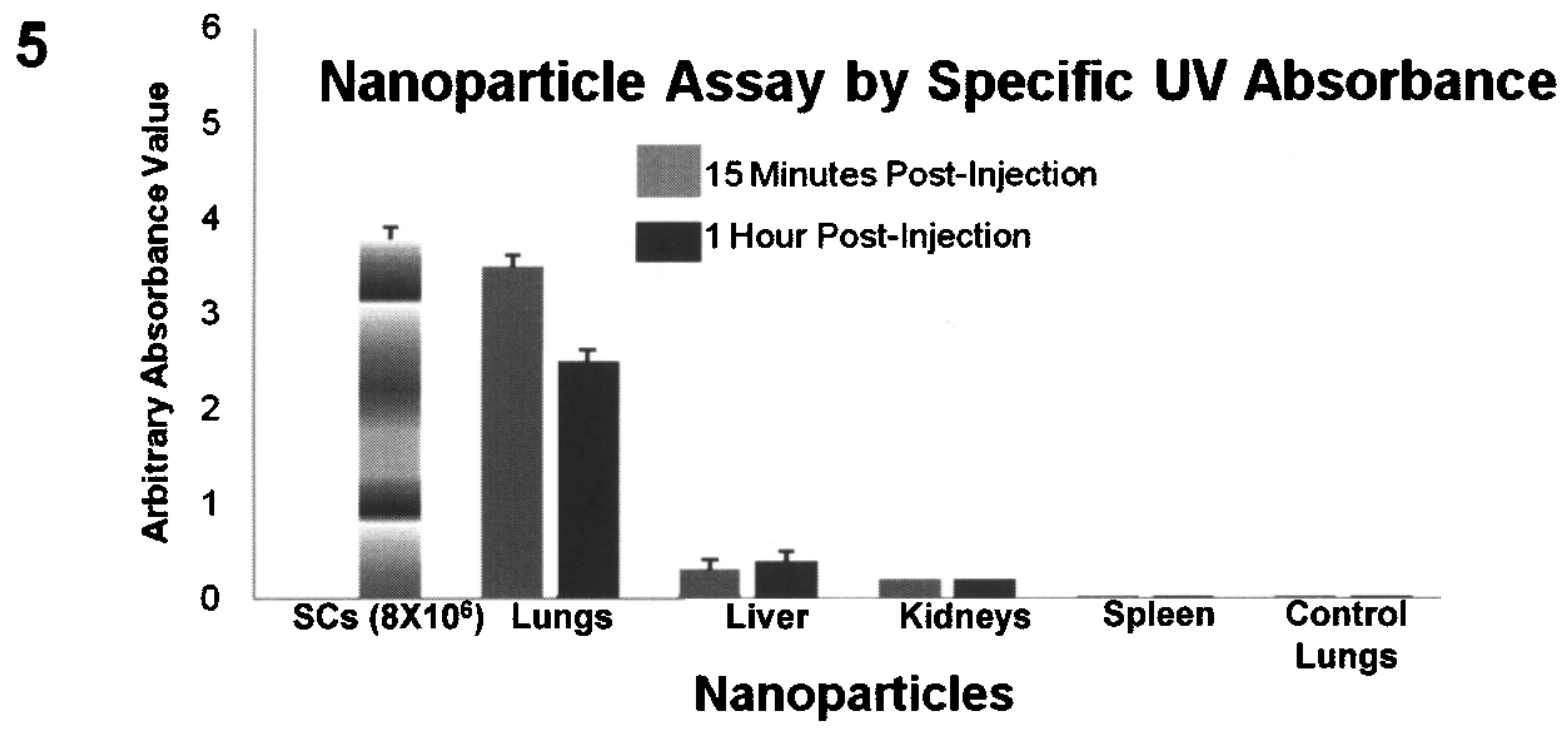

Figure 5. Absorbance (UV at $488 \mathrm{~nm}$ ) generated from FITC-labeled nanoparticles in preloaded rat Sertoli cells (SCs) in vitro $(8 \times$ $10^{6}$ ), in tissues (Lung SNAP inj., Liver, Kidneys, and Spleen) from mice injected with preloaded SCs $\left(8 \times 10^{6}\right)$ collected 15 min $(n=4)$ and $1 \mathrm{~h}$ postinjection $(n=4)$, and from lungs (Lung Control) from control mice $(n=4)$. There was no label detection in spleens or control lungs. 


\section{DISCUSSION}

Results showed that following IV injection, via the mouse lateral tail vein, prelabeled isolated rat SCs preloaded with chitosan nanoparticles became entrapped in the host's deep lung and were distributed around alveoli in the perialveolar sac pulmonary vasculature, and that intact SCs were not detected in other tissues and organs such as the kidneys, thymus, and liver. At 15 min postinjection, the prelabeled, preloaded SCs appeared intact in the lung based on the unique florescent imaging of these cells; and they appeared to occupy a perialveolar sac distribution throughout the organ including the lower lung. Also at 15 min postinjection, $92 \%$ of the labeled nanoparticle load originally contained in the injected SCs was present in the lungs with some minimal label detection in the liver and kidney, but absent in other tissues and organs assayed. However, at $1 \mathrm{~h}$ postinjection, there was no evidence of intact SCs in the lungs, although the volume of nanoparticles in the lungs remained at $\sim 70 \%$ of the total load delivered. The absence and apparent lysis of intact SCs in the lungs was supported by the electron microscopic detection of cellu- lar debris in pulmonary capillaries and the presence of liberated nanoparticles. Based on this and the detection and quantification of the nanoparticle-specific label in the lungs, these results indicate that the IV-injected rat SCs carried and then effectively delivered most of the preloaded nanoparticles into and throughout the deep lung.

Delivery of the anti-inflammatory compound curcumin to the deep lung by SNAP was tested in an animal model of pulmonary inflammation. Following 24 h postinjection of SCs preloaded with curcumin-coupled nanoparticles, all tissue sections from all treated OVA-challenged mice $(n=7)$ showed no evidence of airway or perivascular inflammation whereas all untreated OVAchallenged mice $(n=7)$ retained overt perivascular pulmonary inflammation. This pilot animal experiment to test the utility of the SNAP drug delivery resulted in a conspicuous therapeutic outcome, indicating the efficient delivery of curcumin to the deep lung. Although there was a conspicuous and rapid therapeutic response to the SNAP delivery of curucmin, quantification of the observed therapeutic effect, safe dosing parameters, and a more in-depth study are clearly necessary to better verify and define these observations.

\section{6}

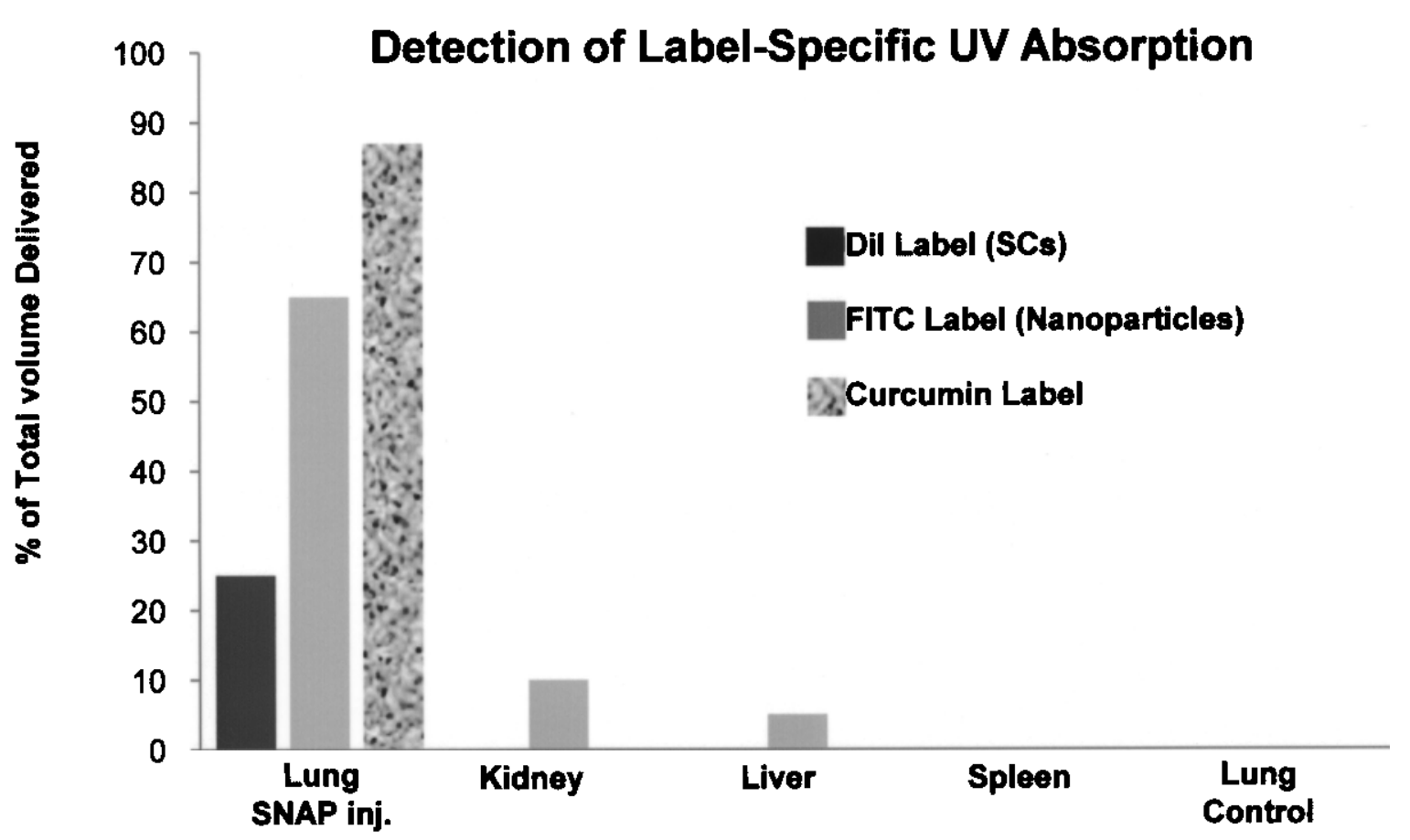

Figure 6. Percent of total of DiI label (Sertoli cells, UV at $553 \mathrm{~nm}$ ) and FITC label (nanoparticles, UV $488 \mathrm{~nm}$ ) $1 \mathrm{~h} \mathrm{following} \mathrm{IV}$ injection of preloaded, prelabeled rat Sertoli cells (SCs) in the mouse host, and percent of total curcumin label (UV at $420 \mathrm{~nm}$ ) 24 $\mathrm{h}$ following IV injection of preloaded rat SCs into the mouse host. Following $1 \mathrm{~h}$ postinjection only $26 \%$ of SC label was detected in the mouse lungs and no SC label was detected in other organs and tissues tested, whereas $65 \%$ of the delivered nannoparticles was detected in the lungs with some detection in the kidney (10\%) and liver (5\%). Twenty-four hours postinjection, $87 \%$ of the curcumin label was detected in the lungs with no detection in other tissues and organs tested. None of the labels were detected in uninjected control mouse lungs. 

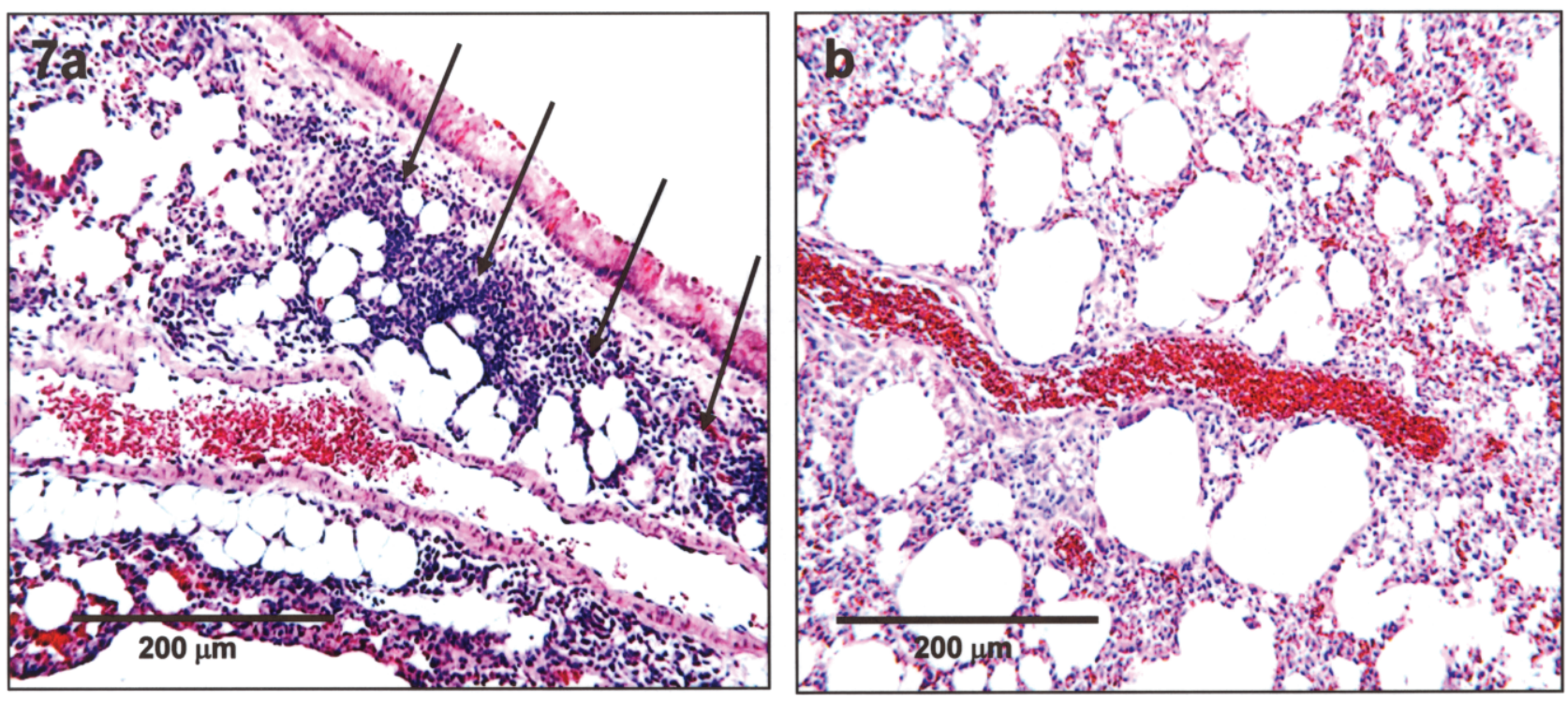

Figure 7. Light microscopic images of sectioned lung tissues from (a) an untreated OVA-challenged mouse and (b) an OVAchallenged mouse $24 \mathrm{~h}$ following injection of SCs preloaded with curcumin-coupled nanoparticles. Lung tissue from the untreated OVA-challenged mouse showed extensive perivascular inflammation as indicated in (a) by the presence of inflammatory cells (arrows). In all seven SC-injected mice (SNAP delivery of curcumin), there was no evidence of pulmonary inflammation based on the absence of inflammatory cell types in the tissue sections, as illustrated in (b). H\&E: $4 \mu \mathrm{m}$.

Nanoparticles such as those constructed of chitosan and utilized in this project have been found to be useful carriers for sustained drug release for up to $96 \mathrm{~h}(12,13)$ and there are currently several administration routes for their pulmonary delivery to the lungs $(12,13,21,22)$. However, by this route, optimal distribution of therapeutic nanoparticles to the entire airway, especially the deep lung, can be limited by deposition in the oropharynx, inappropriate breathing patterns, particle size, particle aggregation, and their exhalation after inspiration $(2,11$, 21). The SNAP delivery of drugs can overcome these limitations, therefore optimizing high-density delivery and retention of drugs into and throughout the organ without accumulation in other organ systems. Because isolated SCs aggressively phagocytose nanoparticles, are self-immunoprotective $(5,20)$, and have not been shown to elicit an inflammatory response by the host following transplantation $(8,19)$, results of this study indicated that the utilization of SNAP drug delivery offers a safe and effective therapeutic modality for the targeted delivery of drug-specific nanoparticles to the deep lung by this unique cell type.

The ability to easily and quickly target just the lungs with an optimal amount of drug, especially to the perialveolar sac region, without evidence of systemic contamination is remarkable and not yet obtainable by other methodologies. As illustrated by the results of the current project, the use of preloaded SCs to deliver therapeutic nanoparticles to the lungs by introducing the cells into the peripheral vasculature, which then go directly to the pulmonary vasculature, may provided a effective alternative to current methodologies and overcome the limitations inherent in the pulmonary delivery route. The delivery of $87 \%$ of the curcumin load to and throughout the lungs and especially the deep lung by SNAP delivery is a significant advantage in drug utilization, particularly in situations where airway delivery is urgent and limited by severe airway obstruction or interstitial edema. These data suggest that SNAP delivery may provide alternative means to deliver high local concentrations of other agents such as immunosuppressive drugs for lung transplantation therapy. Additional utilization of this methodology, when fully verified, could be of benefit in conditions such as ARDS, status asthmaticus, pulmonary hypertension, posttransplantation complications following lung transplantation where inhaled or oral drug delivery is impractical, lung cancer, or where systemic delivery of drugs induces unacceptable drug toxicity.

\section{REFERENCES}

1. Amidi, M.; Romeijn, S. G.; Borchard, G.; Junginger, H. E.; Hennink, W. E.; Jiskoot, W. Preparation and characterization of protein-loaded $\mathrm{N}$-trimethyl chitosan nanoparticles as nasal delivery system. J. Control. Release 11(12):107-116; 2006.

2. Azarmi, S.; Roa, W. H.; Lobenberg, R. Targeted delivery of nanoparticles for the treatment of lung diseases. Adv. Drug Deliv. Rev. 60:863-875; 2008.

3. Cameron, D. F.; Muffly, K. E. Hormonal regulation of 
spermatid binding to Sertoli cells in vitro. J. Cell Sci. 100: $523-533 ; 1991$.

4. Corkery, K. Inhalable drugs for dystemic therapy. Respir. Care 45(7):831-835; 2000

5. Dufour, J.; Hamilton, M.; Rajotte, R.; Korbutt, G. Neonatal procine Sertoli cells inhibit human natural antibodymediated lysis. Biol. Reprod. 72:1224-1231; 2005.

6. Dufour, J. M.; Rajotte, R. V.; Korbutt, G. S.; Emerich, D. F. Harnessing the immunomodulatory properties of Sertoli cells to enable xenotransplantation in type I diabetes. Immunol. Invest. 32:275-297; 2003.

7. Edwards, D. A.; Ben-Jebria, A.; Langer, R. Recent advances in pulmonary drug delivery using large, porous inhaled particles. J. Appl. Physiol. 85:379-385; 1998.

8. Emerich, D. F.; Hemendinger, R.; Halberstadt, C. R. The testicular-derived Sertoli cell: Cellular immunoscience to enable transplantation. Cell Transplant. 12:335-349; 2003.

9. Golat, B.; Cameron, D. F. Sertoli cells enhance formation of capillary-like structures in vitro. Cell Transplant. 17: 1135-1144; 2008

10. Jain, D.; Banergee, R. Comparison of ciprofloxacin hydrochloride-loaded protein, lipid, and chitosan nanoparticles for drug delivery. J. Biomed. Mater. Res. B Appl. Biomater. 86B(1):105-112; 2007.

11. Kleinstreuer, C.; Zhang, Z.; Donohue, J. F. Targeted drugaerosol delivery in the human respiratory system. Annu. Rev. Biomed. Eng. 10:1-26; 2008.

12. Kumar, M.; Kong, X.; Behera, A.; Hellermann, G.; Lockey, R.; Mohapatra, S. Chitosan IFN- $\gamma$-pDNA nanoparticle (CIN) therapy for allergic asthma. Genet. Vaccines Ther. 1(1):3; 2003.

13. Lalor, C. B.; Hickey, A. J. Generation and characterization of aerosols for drug delivery to the lungs. In: Adjei, A.; Gupta, P. K., eds. Inhalation and delivery of therapeutic peptides and proteins. New York: Marcel Dekker; 1997:235-276.

14. Leach, C.; Colice, G. L.; Luskin, A. Particle size of inhaled corticosteroids: Does it matter? J. Allergy Clin. Immunol. 124(6 Suppl.):S88-S93; 2009.

15. Malgorzata, S.; Vandamme, T.; Sokolowski, A. Nanocarriers as pulmonary drug delivery systems to treat and to diagnose respiratory and non respiratory diseases. Int. J. Nanomed. 3(1):1-19; 2008.
16. Newman, S. P. Inhaler treatment options in COPD. Eur. Respir. Rev. 14:102-108; 2005.

17. O'Riordan, T. G.; Palmer, L. B.; Smaldone, G. C. Aerosol deposition in mechanically ventilated patients. Optimizing nebulizer delivery. Am. J. Respir. Crit. Care Med. 149: 214-219; 1994.

18. Patton, J. S. Inhalation delivery of therapeutic peptides and proteins. J. Aerosol Med. 12:45-46; 1999.

19. Shamekh, R.; Dagani, N.; Saporta, S.; Pascual, J.; Sanberg, P. R.; Cameron, D. F. Sertoli cells induce systemic donorspecific tolerance in xenogenic transplantation model. Cell Transplant. 15(1):45-53; 2006.

20. Sipone, S.; Simmen, K. C.; Lord, S. J.; Motyka, B.; Ewen, C.; Shostak, I.; Rayat, G. R.; Dufour, J. M.; Korbutt, G. S.; Rajotte, R. V.; Bleackley, C. Identification of a novel human granzyme B inhibitor secreted by cultured Sertoli cells. J. Immunol. 177:5051-5058; 2006.

21. Sung, J. C.; Pulliam, B. L.; Edwards, D. A. Nanoparticles for drug delivery to the lungs. Trends Biotechnol. 25(12): 563-570; 2007.

22. Temelkovski, J.; Hogan, S. P.; Shepherd, D. P.; Foster, P. S.; Kumar, R. K. An improved murine model of asthma: Selective airway inflammation, epithelial lesions and increased methacholine responsiveness following chronic exposure to aerosolised allergen. Thorax 10:849$856 ; 1998$.

23. Thompson, P. J. Drug delivery to the small airways. Am. J. Respir. Crit. Care Med. 157(5 Pt. 2):S199-202; 1998.

24. Weggman, M. Animal models of chronic experimental asthma-strategies for the identification of new therapeutic targets. J. Occup. Med. Toxicol. 3(Suppl. 1):S4; 2008.

25. Yang, H.; Al-Jazaeri, A.; Wright, R. The immunoprotective effects of Sertoli encapsulated islets xengrafts is not dependent upon FAS ligand expression. Cell Transplant. $11: 788-801 ; 2002$.

26. Yang, W.; Peters, J. I.; Williams, R. O. Inhaled nanoparticles-a current review. Int. J. Pharm. 356:239-247; 2008.

27. Zhang, H.; Oh, M.; Allen, C.; Kumacheva, E. Monodisperse chitosan nanoparticles for mucosal drug delivery. Biomacromolecules 5(6):2461-2468; 2004. 